\title{
AN EXAMPLE CONCERNING PARTS AND NEWTONIAN CAPACITY ${ }^{1}$
}

\author{
JAMES LI-MING WANG
}

\begin{abstract}
We prove the following theorem: Let $\phi$ be an admissible function with $\phi\left(0^{+}\right)=0$ and $p$ a nonnegative integer. Then there is a compact set $X$ in the plane and $x \in X$ such that $x$ is a nonpeak point of $R(X)$,

$$
\sum 2^{(p+1) n} \phi\left(2^{-n}\right)^{-1} \mathcal{C}\left(A_{n}(x) \backslash P(x)\right)<\infty,
$$

while $\Sigma 2^{(p+1) n} \phi\left(2^{-n}\right)^{-1} \gamma\left(A_{n}(x) \backslash X\right)=\infty$, where $A_{n}(x)=\left\{2^{-n-1}<\mid z-\right.$ $\left.x \mid<2^{-n}\right\}, P(x)$ denotes the Gleason part of $x, \gamma$ the analytic capacity and $\mathcal{C}$ the Newtonian capacity.
\end{abstract}

Let $X$ be a compact subset of the complex plane. We denote by $R(X)$ the uniform closure of all rational functions having no poles on $X$. For $x, y \in X$, the Gleason distance is

$$
\|y-x\|=\sup \{|f(y)-f(x)|: f \in R(X),\|f\|<1\} .
$$

The Gleason part of $x$ is $P(x)=\{\|y-x\|<2\}$. We set $P(x, a)=\{\|y-x\|$ $<a\}$, the Gleason ball about $x$ with radius $a$.

In [3], O'Farrell gives a necessary condition in terms of Newtonian capacity $\mathcal{C}$ for a point $x \in X$ to be a nonpeak point of $R(X)$ or $R(X)$ admits a $p$ th order bounded point derivation at $x$, as follows:

THEOREM. (I) If $x$ is a nompeak point of $R(X)$ and $a>0$ then

$$
\sum 2^{n} \mathcal{C}\left(A_{n}(x) \backslash P(x, a)\right)<\infty,
$$

where $A_{n}(x)=\left\{2^{-n-1}<|z-x|<2^{-n}\right\}$ and $\mathcal{C}$ denotes Newtonian capacity.

(II) If $R(X)$ admits a pth order bounded point derivation at $x$ and $a>0$, then

$$
\sum 2^{(p+1) n} \mathcal{C}\left(A_{n}(x) \backslash P(x, a)\right)<\infty .
$$

The converse of $(\mathrm{I})$ is trivial, since if $x$ is a peak point, then $P(x, a)=\{x\}$. O'Farrell [3] also points out that $\mathcal{C}$ may not be replaced by analytic capacity $\gamma$ in this theorem.

One might conjecture that the converse is true in general. This note is to show this conjecture may not be true if $P(x, a)$ is replaced by $P(x)$.

An admissible function $\phi$ is a positive nondecreasing function on the interval $(0, \infty)$ such that $\psi(r)=r / \phi(r)$ is also nondecreasing, with $\psi\left(0^{+}\right)=0$.

Melnikov's theorem (see [2], e.g.) states that a point $x$ in a compact set $X$ is

Received by the editors March 9, 1978 and, in revised form, August 21, 1978.

AMS (MOS) rubject classifications (1970). Primary 30A82, 30A44.

Key words and phrases. Gleason part, Newtonian capacity, analytic capacity.

${ }^{1}$ Research supported in part by NSF Grant \#MCS 77-02208. 
a peak point for $R(X)$ if and only if $\Sigma 2^{n} \gamma\left(A_{n}(x) \backslash X\right)<\infty$. Hallstrom's criterion [2] generalizes that $R(X)$ admits a $p$ th order bounded point derivation at $x$ if and only if

$$
\sum 2^{(p+1) n} \gamma\left(A_{n}(x) \backslash X\right)<\infty .
$$

With the notion of an admissible function, $\phi(r)=r^{\alpha}, 0<\alpha<1$, for instance, Wang [5] and O'Farrell [4] discuss the bounded point derivation of fractional order in terms of analytic capacity. Thus it is not hard to see O'Farrell's theorem in a generalized form: if $\Sigma 2^{(p+1) n} \phi\left(2^{-n}\right)^{-1} \gamma\left(A_{n}(x) \backslash X\right)<\infty$ then $\Sigma 2^{(p+1) n} \phi\left(2^{-n}\right)^{-1} \mathcal{C}\left(A_{n}(x) \backslash P(x, a)\right)<\infty$ for any admissible function $\phi$.

Now we state our theorem in a generalized form.

THEOREM. Let $\phi$ be an admissible function with $\phi\left(0^{+}\right)=0$ and $p$ a nonnegative integer. Then there is a compact set $X$ and $x \in X$ such that $x$ is a nompeak point of $R(X)$.

$$
\sum 2^{(p+1) n} \phi\left(2^{-n}\right)^{-1} \mathcal{C}\left(A_{n}(x) \backslash P(x)\right)<\infty,
$$

while $\Sigma 2^{(p+1) n} \phi\left(2^{-n}\right)^{-1} \gamma\left(A_{n}(x) \backslash X\right)=\infty$.

Proof. Let $a_{n}=\frac{3}{4} 2^{-n}$, and choose $\rho_{n}>0$ so that $\Sigma 2^{n} \rho_{n}<\infty$, but $\Sigma 2^{(p+1) n} \phi\left(2^{-n}\right)^{-1} \rho_{n}=\infty$. Let $D_{n}$ be the open disk with center $a_{n}$ and radius $\rho_{n}$. Let $I_{n}$ be the interval $\left[a_{n}-\frac{1}{2} \rho_{n}, a_{n}+\frac{1}{2} \rho_{n}\right]$. Then $\mathcal{C}\left(I_{n}\right)=0$ (see [1], [6]). Since Newtonian capacity is monotone, subadditive and regular [6], we may choose an open $U_{n}$ with $I_{n} \subset U_{n}, \bar{U}_{n} \subset D_{n}$, such that

$$
\bigodot\left(\bar{U}_{n}\right)<n^{-2} 2^{-(p+1) n} \phi\left(2^{-n}\right) .
$$

Put $X=\bar{\Delta}(0,1) \backslash \cup{ }_{1}^{\infty} U_{n}$. Then $A_{n}(0) \backslash X=U_{n}$, so $\gamma\left(A_{n}(0) \backslash X\right)<\gamma\left(D_{n}\right)=$ $\rho_{n}$, whence $\Sigma 2^{n} \gamma\left(A_{n}(0) \backslash X\right)<\Sigma 2^{n} \rho_{n}<\infty$, so 0 is a nonpeak point of $R(X)$, by Melnikov's theorem. It follows [3] that the part $P$ of 0 contains the interior of $X$, so that $A_{n}(0) \backslash P \subset \bar{U}_{n}$. Thus

$$
\sum 2^{(p+1) n} \phi\left(2^{-n}\right)^{-1} \mathcal{C}\left(A_{n}(0) \backslash P\right)<\sum 2^{(p+1) n} \phi\left(2^{-n}\right)^{-1} \mathcal{C}\left(\bar{U}_{n}\right)<\sum n^{-2}<\infty,
$$

while

$$
\begin{aligned}
\sum 2^{(p+1) n} \phi\left(2^{-n}\right)^{-1} \gamma\left(A_{n}(0) \backslash X\right) & >\sum 2^{(p+1) n} \phi\left(2^{-n}\right)^{-1} \gamma\left(I_{n}\right) \\
& =\frac{1}{4} \sum 2^{(p+1) n} \phi\left(2^{-n}\right)^{-1} \rho_{n}=\infty
\end{aligned}
$$

(see [1]).

REMARK. It can be shown that the series $\Sigma 2^{(p+1) n} \phi\left(2^{-n}\right)^{-1} C\left(A_{n}(0) \backslash\right.$ $P(0, a))$ diverges for any $a, 0<a<2$, for all compact sets $X$ constructed in the theorem. So to give a counterexample to the converse of O'Farrell's result for $a<2$, it appears that some other compact set $X$ is needed. We are grateful to Bernt Oksendal for his helpful communications. 


\section{REFERENCES}

1. J. Garnett, Analytic copacity and measure, Lecture Notes in Math., vol. 297, Springer-Verlag. Berlin, 1972.

2. A. Hallstrom, On bounded point derivations and analytic capacity, J. Functional Analysis 4 (1969), 153-165.

3. A. O'Farrell, Density of parts of algebras on the plane, Trans. Amer. Math. Soc. 196 (1974), $403-414$

4. Analytic capacity and equicontinuity (preprint).

5. J. Wang, Modules of approximate contimuity for $R(X)$, Math. Scand. 34 (1974), 219-225.

6. J. Wermer, Potential theory, Lecture Notes in Math., vol. 408, Springer-Verlag, Berlin, 1974.

Department of Mathibutics, University of Alabama, Universtty, Alabaka 35486 